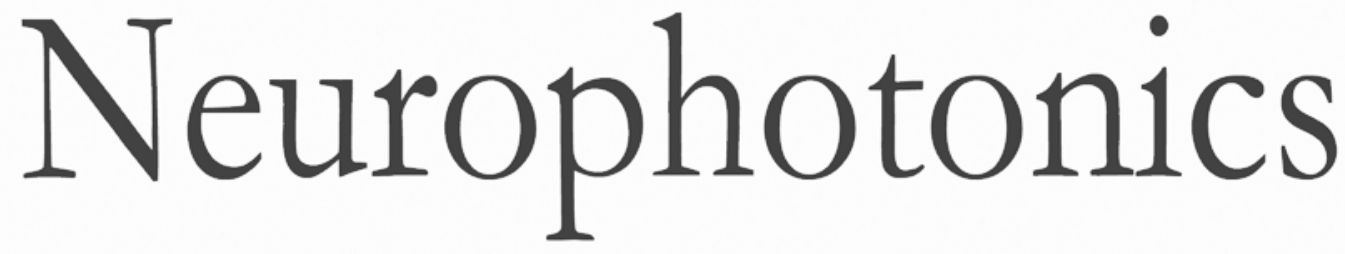

\title{
Using prerecorded hemodynamic response functions in detecting prefrontal pain response: a functional near-infrared spectroscopy study
}

Ke Peng

Meryem A. Yücel

Christopher M. Aasted

Sarah C. Steele

David A. Boas

David Borsook

Lino Becerra 


\title{
Using prerecorded hemodynamic response functions in detecting prefrontal pain response: a functional near-infrared spectroscopy study
}

\author{
Ke Peng, ${ }^{a, b, c, *}$ Meryem A. Yücel, ${ }^{c}$ Christopher M. Aasted, ${ }^{a, b, c}$ Sarah C. Steele, ${ }^{a, b, c}$ \\ David A. Boas, ${ }^{c, d}$ David Borsook, ${ }^{a, b, c}$ and Lino Becerra ${ }^{a, b, c}$ \\ aHarvard Medical School, Center for Pain and the Brain, Boston, Massachusetts, United States \\ ${ }^{b}$ Boston Children's Hospital and Harvard Medical School, Department of Anesthesiology, Perioperative and Pain Medicine, Boston, \\ Massachusetts, United States \\ 'Massachusetts General Hospital and Harvard Medical School, MGH/HST Athinoula A. Martinos Center for Biomedical Imaging, \\ Department of Radiology, Charlestown, Massachusetts, United States \\ ${ }^{\mathrm{d} B o s t o n}$ University, Boston University Neurophotonics Center, Boston, Massachusetts, United States
}

\begin{abstract}
Currently, there is no method for providing a nonverbal objective assessment of pain. Recent work using functional near-infrared spectroscopy (fNIRS) has revealed its potential for objective measures. We conducted two fNIRS scans separated by $30 \mathrm{~min}$ and measured the hemodynamic response to the electrical noxious and innocuous stimuli over the anterior prefrontal cortex (aPFC) in 14 subjects. Based on the estimated hemodynamic response functions (HRFs), we first evaluated the test-retest reliability of using fNIRS in measuring the pain response over the aPFC. We then proposed a general linear model (GLM)-based detection model that employs the subject-specific HRFs from the first scan to detect the pain response in the second scan. Our results indicate that fNIRS has a reasonable reliability in detecting the hemodynamic changes associated with noxious events, especially in the medial portion of the aPFC. Compared with a standard HRF with a fixed shape, including the subject-specific HRFs in the GLM allows for a significant improvement in the detection sensitivity of aPFC pain response. This study supports the potential application of individualized analysis in using fNIRS and provides a robust model to perform objective determination of pain perception. ๑ 2017 Society of Photo-Optical Instrumentation Engineers (SPIE) [DOI: 10.1117/1.NPh.5.1.011018]
\end{abstract}

Keywords: hemodynamic response function; pain; test-retest reliability; anterior prefrontal cortex; detection sensitivity; near-infrared spectroscopy.

Paper 17068SSRR received Apr. 6, 2017; accepted for publication Sep. 26, 2017; published online Oct. 16, 2017.

\section{Introduction}

The development of an objective measure of pain/nociception would be beneficial in many clinical situations including pain assessment in those not able to provide verbal self-reports, measures for evaluation of treatment efficacy, or measures of nociception/ongoing pain during the perioperative period. Recent studies have shown the potential of using functional nearinfrared spectroscopy (fNIRS) in such conditions, given its advantages in cost, robustness, and portability. ${ }^{1,2}$ NIRS is a noninvasive neuroimaging technique that is able to provide longterm measures of cortical hemodynamics [i.e., through measure of the changes of oxygenated hemoglobin $(\mathrm{HbO})$ and deoxygenated hemoglobin (HbR) concentrations]. ${ }^{3-5}$ Previous work using fNIRS in studying pain mainly delineated a significant deoxygenation process (normally a decrease in $\mathrm{HbO}$ concentration) in the anterior prefrontal cortex (aPFC) in response to noxious stimuli, including cutaneous pain, ${ }^{6-8}$ tooth pain, ${ }^{9}$ and visceral pain. ${ }^{10,11}$ Such observations are in line with recent reports of deactivations in aPFC blood oxygenation level-dependent (BOLD) signals following pain using functional magnetic resonance imaging (fMRI). ${ }^{12-15}$ Our previous fNIRS studies reported that the hemodynamic response to noxious stimuli

*Address all correspondence to: Ke Peng, E-mail: Ke. Peng@childrens.harvard .edu was stronger than the response to innocuous stimuli with regard to peak amplitude changes ${ }^{6}$ and that the $\mathrm{HbO}$ decrease is most strongly present in the medial portion of the aPFC. ${ }^{7}$

Several difficulties remain regarding using the defined feature of the aPFC response (viz., the amplitude of $\mathrm{HbO}$ decrease) to detect pain in individual cases including: (1) the hemodynamic response to pain seems to show habituation with repetitive painful stimuli, leading to an insignificant difference in the response amplitude between innocuous stimuli and repeated noxious stimuli; ${ }^{6,7}$ (2) distinct patterns in the shape of the hemodynamic response function (HRF) to pain (in terms of e.g., activations or deactivations, the peak/nadir time, dispersion, and undershoot time) have also been observed across different subjects, brain regions, and types of pain. In addition to the deactivations, multiple studies using fNIRS also reported pain-induced activations in the aPFC, largely in the lateral portion; ${ }^{16-19}$ and (3) while the aPFC hemodynamic changes associated with pain were shown to be bilateral in some studies, others observed predominantly left-lateralized responses. ${ }^{9,10}$ These results suggest using personalized analysis in the detection of the brain response to pain to properly characterize the signal.

In the present study, we sought to use fNIRS to detect aPFC response in healthy individuals with prerecorded, personalized HRF to nociception. The subjects involved in this study

2329-423X/2017/\$25.00 @ 2017 SPIE 
(a)

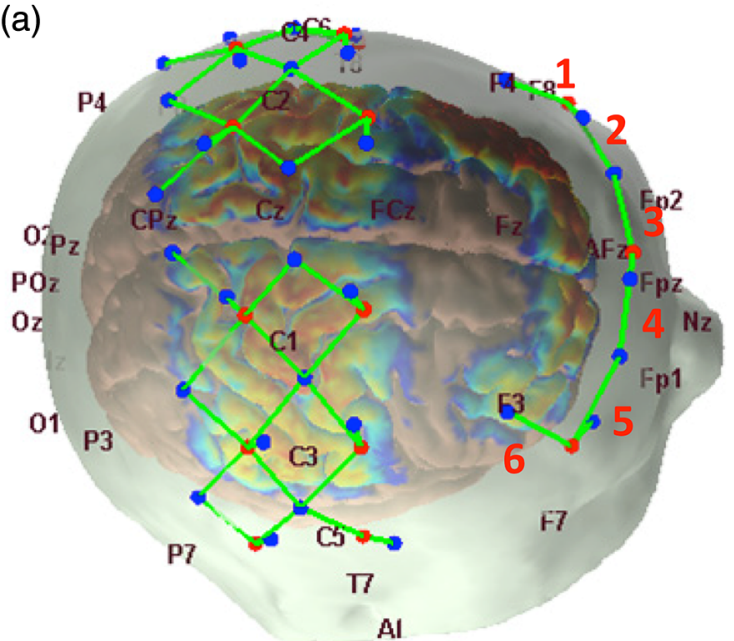

(b)

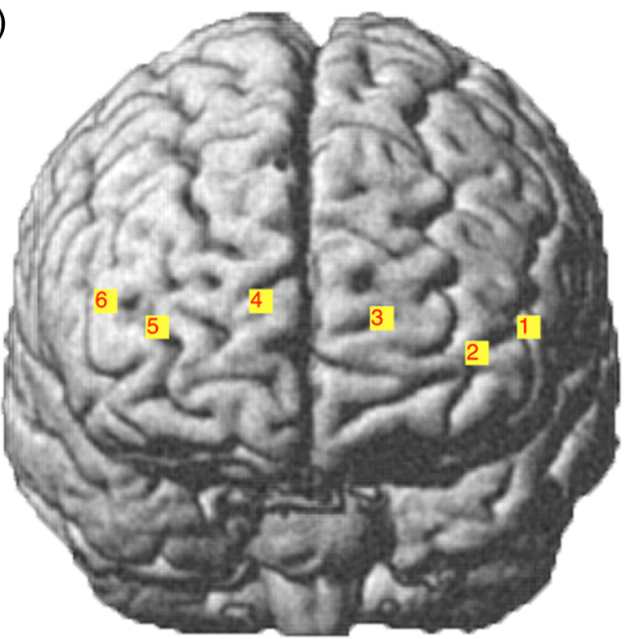

Fig. 1 (a) The arrangement of fNIRS optodes and the corresponding sensitivity profile. Image is adapted from our prior study. ${ }^{21}$ In this study, we focus on detecting pain response from the six prefrontal channels (marked in red). (b) The coregistration of the six prefrontal channels onto a T1 MRI template. The coordinates of the prefrontal channels are obtained based on an average of the locations on the 14 subjects.

underwent two 3-min fNIRS scans (a prescan and a detection scan) separated by $30 \mathrm{~min}$ of resting period. In the first part of this study, we evaluate the feasibility of using prerecorded HRF to detect subsequent painful/nonpainful events by quantitatively estimating the test-retest reliability of the fNIRS-measured HRF to noxious and innocuous stimuli in the aPFC between the prescan and the detection scan. Based on the results, we then propose a detection model under a general linear model (GLM) framework, which employs the subject-specific HRF estimated from the prescan to detect the aPFC response to the two types of stimuli in the detection scan. The GLM has been widely applied in the detection of evoked brain hemodynamic changes [see Monti ${ }^{20}$ for a review]. Two approaches are compared in the current analysis: (a) a standard GLM with the statistical parametric mapping (SPM) canonical HRF (cHRF) and (b) the proposed detection model that employs personalized, prerecorded HRFs. The detection sensitivity of using these two analysis methods to detect aPFC response to noxious and innocuous stimuli is reported. This study suggests that fNIRS is reliable in detecting the prefrontal response associated with pain and proposes a simple but robust individualized pain detection model that can be easily implemented in clinical settings. This approach may potentially be used to provide rapid assessment of pain, e.g., to monitor pain perception on people unable to communicate (such as patients under general anesthesia, babies, stroke patients, and patients with mental disorders) to evaluate the effectiveness of pain treatment.

\section{Methods}

\subsection{Subjects}

This study was approved by the Institutional Review Board of the Massachusetts General Hospital. The study conformed to the ethical standards for human experimentation as defined by the Helsinki Accord and the International Association for the Study of Pain. Sixteen healthy subjects were recruited for this study (right handed, males, ages 19 to 38, mean age $27 \pm 6$ ). Each subject provided written informed consent prior to the scan. Exclusion criteria included a history of neurological trauma or psychiatric disorders and the inability to remain still for six consecutive minutes.

\subsection{Data Acquisition and Experimental Design}

A multichannel continuous wave fNIRS system (CW7, TechEn, Massachusetts) operating at 690- and 830-nm wavelengths was used for this study. Fifteen light emitters and 16 light detectors were mounted on the subject's head, forming 36 30-mm fNIRS channels covering the anterior portion of the prefrontal lobe, as well as the bilateral motor and somatosensory regions [see Fig. 1(a)]. A short separation detector was also installed $8 \mathrm{~mm}$ from each light-emitter to detect signal changes coming from extra cerebral layers (e.g., skin, scalp, and skull).

Prior to the actual scans, we applied $5 \mathrm{~Hz}$ electrical stimulations (Neurotron, Maryland) to each subject's left thumb to determine his level of subjective pain perception. The subject was asked to report ratings as a $3 / 10$ score and a $7 / 10$ score for the perceived sensation, with the $3 / 10$ score being that "the subject should be strongly aware of the stimulus but should not perceive any pain" (innocuous) and the 7/10 score being "the subject should perceive pain, but the pain should be tolerable without breath holding or any retreat actions" (noxious). The intensities of the electrical shocks, which deliver the innocuous and noxious stimuli, were recorded and then used in the actual fNIRS scan.

For each subject, two fNIRS acquisition sessions were conducted: one detection scan and one prescan, which was performed $30 \mathrm{~min}$ prior to the detection scan. Each recording session lasted $\sim 3 \mathrm{~min}$ and contained a randomized sequence of three noxious stimuli and three innocuous stimuli. Both types of electrical stimulations were applied continuously for $5 \mathrm{~s}$ and were separated by $25 \mathrm{~s}$ of resting period.

\subsection{Data Preprocessing and Hemodynamic Response Functions Estimation}

As this study focuses on detecting the aPFC hemodynamic response to pain, only the fNIRS data recorded from the six 
prefrontal channels (see Fig. 1) were used for analysis. Data were preprocessed with the HOMER2 toolbox ${ }^{22}$ implemented in MATLAB (Mathworks, Massachusetts). Please refer to Yücel et al. ${ }^{6}$ for a detailed description of the preprocessing pipeline. In brief, the raw fNIRS signals were first converted into optical density changes and were further transformed to $\mathrm{HbO}$ and $\mathrm{HbR}$ concentration changes using the modified Beer-Lambert law. These hemoglobin concentration changes were then low-passed filtered at $0.5 \mathrm{~Hz}$ with a third-order Butterworth filter. For each of the six normal fNIRS channels, the measured hemoglobin concentration time course $Z$ of a recording session was decomposed with the following deconvolution model: ${ }^{23}$

$Z=R_{N} * \gamma_{N}+R_{I} * \gamma_{I}+D * \gamma_{D}+S * \gamma_{S}+\epsilon$,

where $R_{N}$ and $R_{I}$ represent the hemodynamic changes associated with noxious and innocuous stimuli modeled by the convolution of the stimulus timing $T$ with a series of basis functions $B$ (consecutive Gaussian functions with a standard deviation of $1 \mathrm{~s}$ and their means separated by $1 \mathrm{~s}$ in this study), respectively, $D$ is the modeled drift in the data (polynomial regressors up to the third order), $S$ is the time course from a short separation channel that showed the highest correlation with the time course of the current normal channel (i.e., $Z$ ), $\gamma_{N}, \gamma_{I}, \gamma_{D}$, and $\gamma_{S}$ are the corresponding coefficients, and $\epsilon$ is the error term.

From Eq. (1), the coefficients $\gamma_{N}$ and $\gamma_{I}$ were estimated with a least-square approach, i.e.,

$\hat{\gamma}=\left(A^{T} A\right)^{-1} A^{\mathrm{T}} Z$,

where $A=\left[R_{N}, R_{I}, D, S\right]$ and $\hat{\gamma}=\left[\hat{\gamma}_{N}, \hat{\gamma}_{I}, \hat{\gamma}_{D}, \hat{\gamma}_{S}\right]^{\mathrm{T}}$. The processed time course $Y$ of the session was then reconstructed from $Z$ by removing the drift component and the physiological noises

$Y=Z-D * \hat{\gamma}_{D}-S * \hat{\gamma}_{S}$.

The subject-specific hemodynamic response functions (sHRFs) to noxious stimuli $F_{N}$ and to innocuous stimuli $F_{I}$ of the specific channel were obtained by

$F_{N}=B * \hat{\gamma}_{N} \quad F_{I}=B * \hat{\gamma}_{I}$.

In this study, we focused on the $\mathrm{HbO}$ concentration changes as the recorded $\mathrm{HbR}$ changes were generally noisy and unreliable, which made it difficult to identify the true hemodynamic response to those two types of electrical stimuli.

\subsection{Test-Retest Reliability Evaluation}

We compared the sHRFs of $\mathrm{HbO}$ concentration changes estimated from the two scans (i.e., the prescan and the detection scan) to evaluate the test-retest reliability of the fNIRS-measured aPFC hemodynamic changes associated with noxious and innocuous stimuli. On each channel of each subject, the Spearman's rank correlation coefficient (Spearman's $\rho$ ) was first calculated to test the overall monotonic relationship between the sHRFs from the two scans. In addition to the overall resemblance, we obtained the intraclass correlation coefficients (ICCs) using a two-way random effect analysis of variance model $^{24}$ (with "scan sessions" as the column factor and "subjects" as the row factor) to provide a quantitative measure of the consistency between the main response of the sHRFs (defined in terms of main nadir/peak magnitude and latency) across scans. The main nadir (or peak) of an sHRF was recognized automatically by locating the lowest value (or the highest value if an $\mathrm{HbO}$ increase was present) in the sHRF within 2 to $15 \mathrm{~s}$ after the stimulation onset. The identified nadirs or peaks of the sHRFs were then reviewed manually. To reduce the sensitivity to spurious noise that could create an unusually low nadir (or high peak), the magnitude of a nadir/peak was represented by an average of the sHRF amplitudes over a time window of $2 \mathrm{~s}$ before the nadir/peak to $2 \mathrm{~s}$ after the nadir/peak. Finally, for noxious stimuli, we conducted one-tailed Wilcoxon signed-rank tests between the nadir/peak magnitudes of the sHRFs estimated from the prescan and the detection scan to evaluate the extent of signal habituation in the data. The Wilcoxon signed-rank test is a nonparametric test that requires few assumptions on sample data distribution or variance, making it suitable for testing small sample size data. Therefore, we employed the Wilcoxon signed-rank tests to test the sHRF magnitudes due to the limited number of subjects included in this study.

\subsection{General Linear Model Analysis: Standard Approach and a Detection Model}

The GLM analysis was carried out using the nirs10 MATLAB toolbox $^{25}$ developed based on SPM8 ${ }^{26}$ and NIRS-SPM. ${ }^{27,28}$ For each subject, the $\mathrm{HbO}$ dataset obtained from the detection scan was used as the detection set. The detection process was performed using two approaches, i.e., a standard GLM or the proposed GLM-based detection model.

In the standard GLM approach, the processed channel-wise time course of the measured $\mathrm{HbO}$ concentration changes $Y$ [see Eq. (3)] of the detection scan was expressed with the GLM as follows:

$Y=X_{N} \beta_{N}+X_{I} \beta_{I}+\beta_{0}+\varepsilon$,

where $X_{N}$ and $X_{I}$ are the expected hemodynamic responses to noxious and innocuous stimuli, respectively (computed by convolving the timing of the stimuli with the SPM $8 \mathrm{cHRF}$ ), $\beta_{N}$ and $\beta_{I}$ are the corresponding regression coefficients, $\beta_{0}$ is a constant regressor, and $\varepsilon$ is the error term. The regression coefficient $\hat{\beta}$ was then derived from the least square solution

$\hat{\beta}=\left(X^{\mathrm{T}} X\right)^{-1} X^{\mathrm{T}} Y$,

where $\hat{\beta}=\left[\hat{\beta}_{N}, \hat{\beta}_{I}, \hat{\beta}_{0}\right]^{\mathrm{T}}$. Due to the sparse distribution of the NIRS channels, $\hat{\beta}$ values were spatially interpolated to each pixel of four two-dimensional (2-D) projections (dorsal, frontal, left, and right) using an inhomogeneous interpolation kernel $K .^{27}$ At each pixel, two-tailed $t$-statistic tests were conducted to test the hypothesis that the regression coefficient corresponding to the expected response of noxious $\left(X_{N}\right)$ or innocuous $\left(X_{I}\right)$ stimuli was not significantly different from 0 (i.e., the recorded $\mathrm{HbO}$ response did not change as expected with the presence of noxious or innocuous stimuli)

$$
\left\{\begin{array}{l}
H_{0}: \beta_{s}=0 \\
H_{1}: \beta_{s} \neq 0
\end{array}\right.
$$

where $s=\{N, I\}$. The $t$-statistic at a pixel $l$ was derived by ${ }^{27}$ 

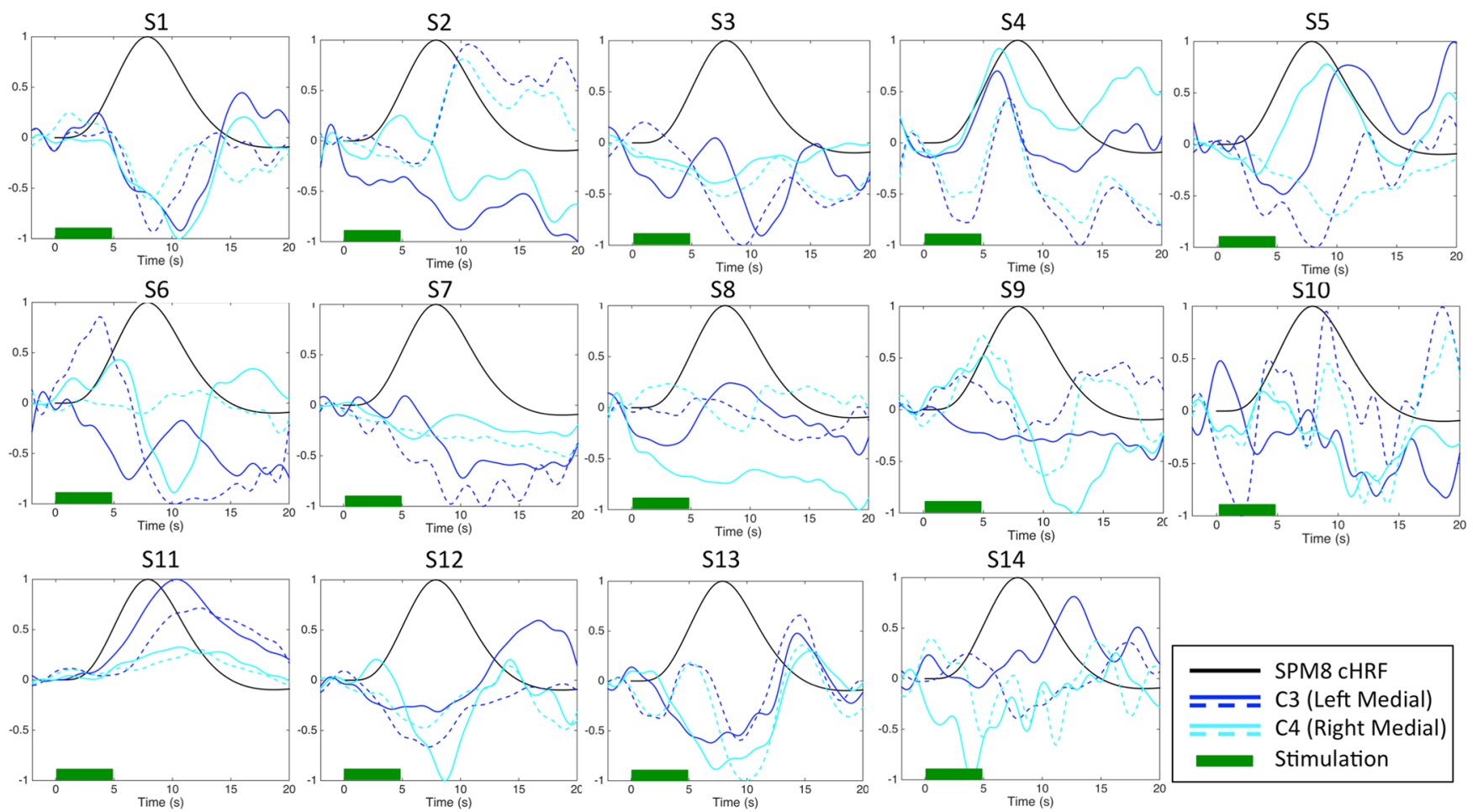

Fig. 2 Medial channels (C3 and C4): comparison of the SPM8 CHRF and the channel-wise sHRFs to noxious stimuli estimated from the prescan (solid curves) and the detection scan (dashed curves). Each panel represents data from a subject.

$t_{s, l}=\frac{\hat{\beta}_{s} K_{l}}{\sqrt{C_{s, l}}}$,

where $C_{s, l}$ is the element in the error covariance matrix of stimulus $s$ corresponding to the pixel $l$ (for details, please refer to Ye et $\mathrm{al}^{27}$ ). The obtained $t$-scores of all the pixels in a $2-\mathrm{D}$ projection were corrected using the Euler characteristic (EC) correction method to yield a family-wise error rate of $p<0.05^{29}$ and were then displayed as $t$-statistical contrast maps.

In the proposed detection model approach, instead of applying a cHRF, we calculated the expected hemodynamic response to noxious and innocuous stimuli with the corresponding subject-specific HRFs obtained from the prescan. To better compare with the GLM results generated with the cHRF (i.e., the standard approach), the sign of the estimated sHRFs was flipped before being passed to the GLM. This is because the main response of the cHRF is depicted as a peak increase, while the main response of sHRFs is usually observed to be $\mathrm{HbO}$ decreases. Therefore, by inverting the sign of the sHRFs, the brain regions that showed positive correlations between the response in the prescan and in the detection scan would be associated with negative $t$-scores, making them easier to be compared with the deactivation clusters detected with the cHRF. The flipped sHRFs from the four aPFC channels $[\mathrm{C} 2, \mathrm{C} 3$, C4, C5, see Fig. 1(b)] were used, respectively, generating four $t$-contrast map sets of each stimulus type (noxious or innocuous) for each subject with a similar GLM approach as described above. The four $t$-contrast map sets of a subject were then combined into one $t$-contrast map set by taking the smallest $t$-score (i.e., the most negative) across the four map sets at each pixel. Adapted from previous fMRI studies, ${ }^{30}$ this procedure was intended to account for the variability in the specific
aPFC regions, which show pain-related response across different subjects.

\subsection{Detection Sensitivity Definition}

Based on the $t$-contrast maps, we compared the sensitivity of using the two detection methods (i.e., the standard GLM and the detection model) in detecting the fNIRS-measured $\mathrm{HbO}$ change associated with noxious and innocuous stimuli. For each subject, we defined positive sensitivity $(\mathrm{SEN}=1)$ for a detection method if we were able to locate at least one cluster of pixels with negative $t$-scores associated with noxious or innocuous stimuli over the aPFC on the $t$-contrast maps generated with the method. The overall sensitivity of a method was estimated by dividing the number of subjects on whom the method was sensitive in the detection by the total number of subjects involved in the analysis.

\section{Results}

\subsection{Comparison of $\mathrm{CHRF}$ and sHRF}

Data of two subjects were excluded from the analysis in the preprocessing step due to excessive noise and motion artifacts. For the remaining 14 subjects, we plotted the sHRFs to noxious stimuli estimated from the two recording sessions in Fig. 2 (medial channels) and Fig. 3 (lateral channels). The sHRFs to innocuous stimuli, as well as the parameter estimates of the nadir/peak magnitude and latency of the sHRFs to both types of stimuli, can be found in the Appendix. In Figs. 2 and 3, the magnitudes of the sHRFs have been normalized so that the highest magnitude is shown as a unit value. Decreases in $\mathrm{HbO}$ concentration associated with noxious stimuli 

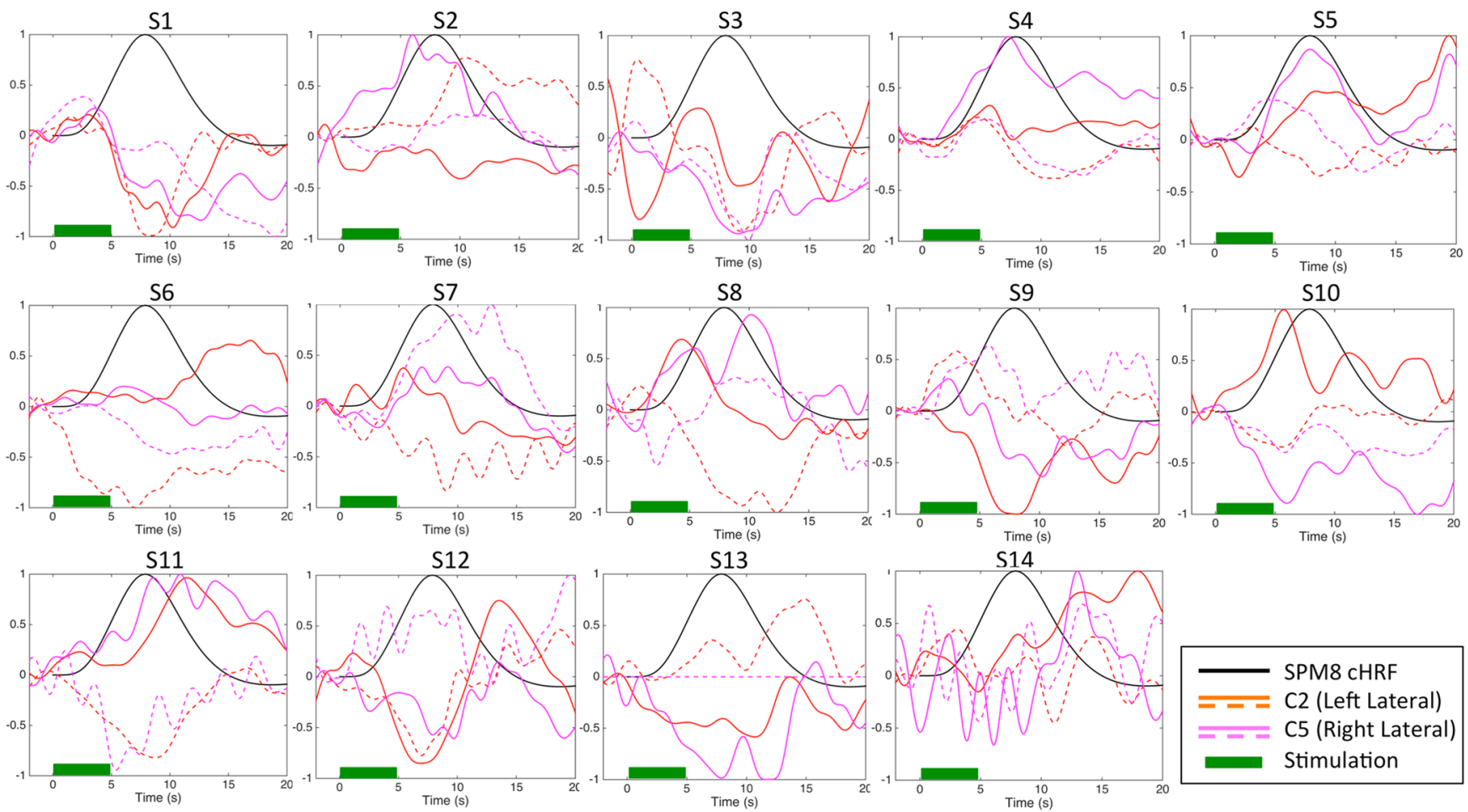

Fig. 3 Lateral channels (C2 and C5): comparison of the SPM8 cHRF and the channel-wise sHRFs to noxious stimuli estimated from the prescan (solid curves) and the detection scan (dashed curves).

were seen in most of the subjects. However, it should be noted that the shape of the response varied from the cHRF with respect to peak latency and peak dispersion. In addition, we also observed $\mathrm{HbO}$ increases in some subjects, especially from the lateral channels (Fig. 3). Interpretation of the observed deactivations and activations is discussed in Sec. 4.3.

\subsection{Test-Retest Reliability of fNIRS-Measured aPFC Response}

We present the ICC and the Spearman's correlation coefficient values of the sHRFs to noxious (Table 1) and innocuous stimuli (Table 2) across the prescan and the detection scan. Along with the ICC values, the $95 \%$ confidence interval, the $F$-score, and the $p$-value were also obtained. In the medial channels (C3 and $\mathrm{C} 4$ ), noxious stimuli were seen to be associated with higher ICC values and Spearman's correlation coefficients when compared with innocuous stimuli. The most reliable measure for noxious stimuli came from the left medial channel (C3), with ICC values for sHRF magnitude and latency being 0.785 and 0.633 , respectively, as well as the Spearman's correlation coefficient being 0.45 after excluding four outlier subjects. The best channel for innocuous stimuli was C4 (right medial), with ICC values of 0.357 and 0.407 for peak/nadir magnitude and latency, respectively. It should also be noted that, for the lateral channels, we obtained a negative ICC value for the nadir/peak magnitude of the sHRFs to noxious or innocuous stimuli. This is due to a larger within-subject variance than between-subject variance in the magnitude dataset, indicating that this measure from the lateral channel was not reliable.

Finally, we compared the nadir/peak magnitude of the sHRFs to noxious stimuli between the prescan and the detection scan to evaluate the extent of habituation of pain signals in our data.
Although we observed magnitude attenuation on some subjects (e.g., subjects \#8 and \#9, see Fig. 2 and Appendix) especially from the medial channels, the difference in the magnitudes did not reach statistical significance $(p$ values $=0.50 / 0.16$ for medial channels $\mathrm{C} 3 / \mathrm{C} 4$ ). This might be explained by the small number of stimuli we delivered to the subjects, as well as the relatively small subject sample size in this study. In addition, the 30-min resting period between the prescan and the detection scan may be enough to eliminate the effect of habituation in some subjects.

\subsection{General Linear Model Results and Detection Sensitivity}

Table 3 provides the GLM results on noxious stimuli of the 14 subjects. The peak $t$-statistical scores and the spatial profiles of the detected clusters with negative $t$-scores using the standard GLM (with the cHRF) or the detection model (with prerecorded sHRFs) are presented, respectively. In 10 of the 14 subjects, we observed a lower peak $t$-score and/or a larger spatial extent of the detected cluster using the detection model. The difference in the peak t-scores between the standard GLM and the detection model was shown to be statistically significant with a one-tailed Wilcoxon signed-rank test at 0.05 testing the null hypothesis that the peak $t$-scores generated with the sHRF model were not lower than those obtained using the $\operatorname{cHRF}(p=0.00098)$.

With the standard GLM approach, statistically significant hemodynamic changes associated with noxious stimuli were located over the aPFC in 2 of the 14 subjects (subjects \#7 and \#11), leading to a detection sensitivity of $14.3 \%$. The sensitivity was improved to $64.3 \%$ using the detection model with prerecorded sHRFs ( 9 of 14 successful cases). Figure 4 presents a case study to further illustrate the detection process. 
Table 1 ICC and Spearman's correlation coefficient values of the sHRFs to noxious stimuli across the prescan and the detection scan. For Spearman's correlation coefficient, the $\rho$-average is from a simple average across all subjects, and the $\rho$-positive is from an averaging process where all negative coefficients were excluded. The number in the parentheses indicates how many subjects were included in the averaging process.

\begin{tabular}{|c|c|c|c|c|}
\hline & C2 (left lateral) & C3 (left medial) & C4 (right medial) & C5 (right lateral) \\
\hline \multicolumn{5}{|c|}{ ICC values } \\
\hline \multicolumn{5}{|c|}{ Peak/nadir magnitude } \\
\hline ICC & -0.429 & $0.785^{\star}$ & $0.660^{*}$ & 0.163 \\
\hline $\mathrm{Cl}$ upper & 0.144 & 0.926 & 0.876 & 0.638 \\
\hline Cl lower & -0.819 & 0.447 & 0.235 & -0.396 \\
\hline$F$-value & 0.414 & 7.795 & 4.860 & 1.390 \\
\hline$p$-value & 0.062 & 0.000367 & 0.00380 & 0.289 \\
\hline \multicolumn{5}{|c|}{ Peak/nadir latency } \\
\hline ICC & 0.014 & $0.633^{*}$ & 0.181 & 0.305 \\
\hline Cl upper & 0.537 & 0.865 & 0.633 & 0.709 \\
\hline Cl lower & -0.547 & 0.179 & -0.356 & -0.207 \\
\hline$F$-value & 1.027 & 4.328 & 1.444 & 1.964 \\
\hline$p$-value & 0.481 & 0.0064 & 0.259 & 0.128 \\
\hline \multicolumn{5}{|c|}{ Spearman's rank correlation coefficient values } \\
\hline$\rho$-average & $0.003 \pm 0.45$ & $0.21 \pm 0.47$ & $0.20 \pm 0.50$ & $0.26 \pm 0.44$ \\
\hline$\rho$-positive & $0.33 \pm 0.25(8 / 14)$ & $0.45 \pm 0.27(10 / 14)$ & $0.42 \pm 0.26(11 / 14)$ & $0.40 \pm 0.28(11 / 13)$ \\
\hline
\end{tabular}

*The ICC estimate was significant under $p<0.05$.

Table 2 ICC and Spearman's correlation coefficient values of the sHRFs to innocuous stimuli across the prescan and the detection scan.

\begin{tabular}{|c|c|c|c|c|}
\hline & C2 (left lateral) & C3 (left medial) & C4 (right medial) & C5 (right lateral) \\
\hline \multicolumn{5}{|c|}{ ICC values } \\
\hline \multicolumn{5}{|c|}{ Peak/nadir magnitude } \\
\hline ICC & 0.384 & 0.332 & 0.357 & -0.061 \\
\hline Cl upper & 0.742 & 0.726 & 0.741 & 0.462 \\
\hline Cl lower & -0.113 & -0.236 & -0.226 & -0.507 \\
\hline$F$-value & 2.349 & 1.952 & 2.038 & 0.874 \\
\hline$p$-value & 0.068 & 0.121 & 0.106 & 0.401 \\
\hline \multicolumn{5}{|c|}{ Peak/nadir latency } \\
\hline ICC & 0.176 & 0.216 & 0.407 & 0.317 \\
\hline $\mathrm{Cl}$ upper & 0.630 & 0.671 & 0.765 & 0.733 \\
\hline Cl lower & -0.359 & -0.354 & -0.163 & -0.302 \\
\hline$F$-value & 1.430 & 1.563 & 2.286 & 1.860 \\
\hline$p$-value & 0.264 & 0.216 & 0.075 & 0.148 \\
\hline \multicolumn{5}{|c|}{ Spearman's rank correlation coefficient values } \\
\hline$\rho$-average & $-0.07 \pm 0.29$ & $0.053 \pm 0.42$ & $0.17 \pm 0.47$ & $0.02 \pm 0.40$ \\
\hline$\rho$-positive & $0.25 \pm 0.15(5 / 14)$ & $0.35 \pm 0.20(8 / 14)$ & $0.45 \pm 0.28(9 / 14)$ & $0.20 \pm 0.18(10 / 13)$ \\
\hline
\end{tabular}


Peng et al.: Using prerecorded hemodynamic response functions in detecting prefrontal pain response...

Table 3 Detected response to noxious stimuli: peak $t$-statistical scores and spatial profiles with the standard GLM approach and with the detection model. For the subjects where no statistically significant clusters could be located, uncorrected $t$-scores are reported and were used in the Wilcoxon signed-rank test to calculate the $p$-value.

\begin{tabular}{|c|c|c|c|c|c|c|}
\hline \multirow[b]{2}{*}{ Subjects } & \multicolumn{3}{|c|}{ Standard approach (cHRF) } & \multicolumn{3}{|c|}{ Detection model (sHRF) } \\
\hline & $\operatorname{Min}(t)$ & Location & SEN & $\operatorname{Min}(t)$ & Location & SEN \\
\hline 1 & -2.4 & Left medial & 0 & -2.4 & Left medial & 0 \\
\hline 2 & NR & & 0 & NR & & 0 \\
\hline 3 & -2.3 & Bilateral medial and lateral & 0 & $-2.9^{*}$ & Bilateral medial & 1 \\
\hline 4 & NR & & 0 & $-2.6^{\star}$ & Right medial & 1 \\
\hline 5 & -2.5 & Bilateral medial & 0 & -2.2 & Right lateral & 0 \\
\hline 6 & -2.0 & Bilateral medial & 0 & $-4.7^{*}$ & Bilateral medial & 1 \\
\hline 7 & $-2.7^{\star}$ & Left lateral & 1 & $-5.8^{*}$ & Bilateral medial and lateral & 1 \\
\hline 8 & NR & & 0 & $-3.4^{*}$ & Bilateral medial and lateral & 1 \\
\hline 9 & NR & & 0 & NR & & 0 \\
\hline 10 & NR & & 0 & $-2.7^{*}$ & Right lateral & 1 \\
\hline 11 & $-3.4^{*}$ & Left medial and right lateral & 1 & $-8.7^{*}$ & Left medial and lateral and right medial & 1 \\
\hline 12 & -2.3 & Left medial and lateral & 0 & $-2.7^{\star}$ & Left medial and lateral & 1 \\
\hline 13 & -1.8 & Left lateral & 0 & -2.4 & Left medial and lateral & 0 \\
\hline 14 & -2.2 & Bilateral medial & 0 & $-2.6^{*}$ & Right lateral & 1 \\
\hline Sensitivity & & & $14.3 \%$ & & & $64.3 \%$ \\
\hline$p$-value & & & & 0.00098 & & \\
\hline
\end{tabular}

Note: NR, no response.

${ }^{*}$ The detected response was statistically significant under EC correction.

(a)

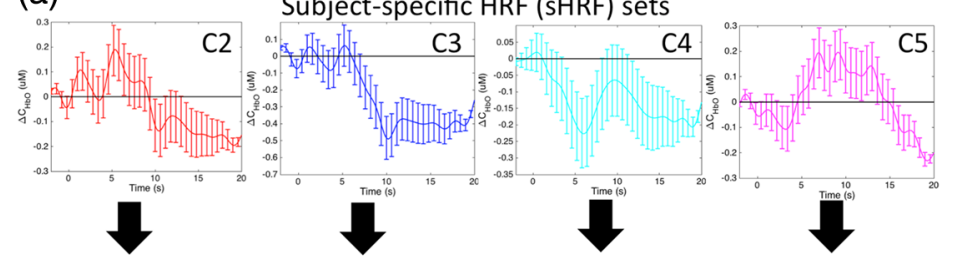

Contrast maps generated with each sHRF
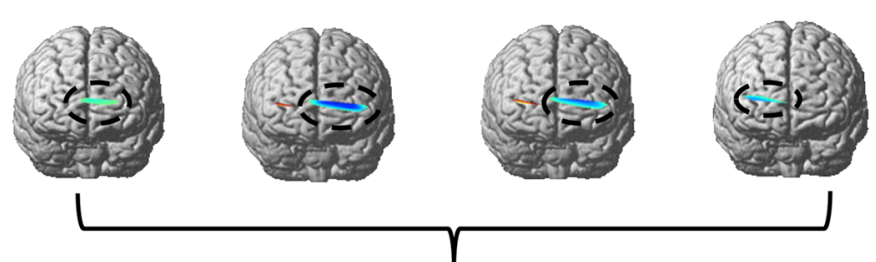

(b)

SPM8 canonical HRF (cHRF)
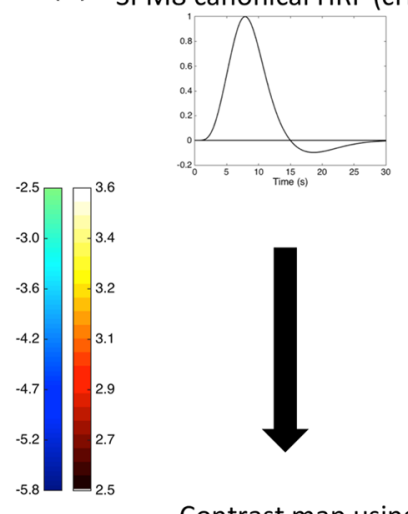

Contrast map using standard GLM (cHRF)

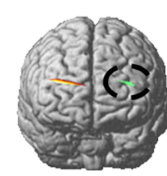

Fig. 4 Case study, subject 7. (a) The GLM-based detection model approach. The top row shows the sHRFs to noxious stimuli estimated from the prescan. Error bars indicate standard error across the three stimulus trials. The middle row depicts the $t$-statistical contrast maps generated with GLM using the four estimated sHRFs, respectively, EC corrected, $p<0.05$. The bottom row shows the final spatial map for detection combined from the above four $t$-map sets. Significant clusters with negative $t$-scores (circled in black) were seen in bilateral medial and lateral portions of the aPFC (detection result: success). (b) The standard GLM approach. The above image shows the SPM8 cHRF. The bottom is the corresponding statistical map using the $\mathrm{cHRF}$, EC corrected, $p<0.05$. A much smaller cluster with less significant negative $t$-scores was seen (mainly in the left lateral aPFC). 
Table 4 Detected response to innocuous stimuli: peak $t$-statistical scores and spatial profiles with the standard GLM approach and with the detection model.

\begin{tabular}{|c|c|c|c|c|c|c|}
\hline \multirow[b]{2}{*}{ Subjects } & \multicolumn{3}{|c|}{ Standard approach (cHRF) } & \multicolumn{3}{|c|}{ Detection model (sHRF) } \\
\hline & $\operatorname{Min}(t)$ & Location & SEN & $\operatorname{Min}(t)$ & Location & SEN \\
\hline 1 & NR & & 0 & $-4.2^{*}$ & Left lateral & 1 \\
\hline 2 & NR & & 0 & $-3.1^{*}$ & Bilateral medial & 1 \\
\hline 3 & $N R$ & & 0 & -1.8 & Left lateral & 0 \\
\hline 4 & $N R$ & & 0 & $-2.8^{*}$ & Right medial & 1 \\
\hline 5 & $-4.6^{*}$ & Left medial & 1 & $-3.7^{\star}$ & Bilateral medial & 1 \\
\hline 6 & NR & & 0 & $-2.8^{\star}$ & Left medial & 1 \\
\hline 7 & NR & & 0 & $-3.3^{*}$ & Right lateral & 1 \\
\hline 8 & $-3.1^{*}$ & Bilateral medial & 1 & $-3.1^{*}$ & Right medial & 1 \\
\hline 9 & NR & & 0 & $-3.1^{*}$ & Right lateral & 1 \\
\hline 10 & -2.1 & Right lateral & 0 & NR & & 0 \\
\hline 11 & -1.8 & Left medial & 0 & -2.0 & Left medial & 0 \\
\hline 12 & $N R$ & & 0 & $-3.2^{*}$ & Bilateral medial and right lateral & 1 \\
\hline 13 & NR & & 0 & -2.0 & Right lateral & 0 \\
\hline 14 & $-3.2^{*}$ & Right medial & 1 & NR & & 0 \\
\hline Sensitivity & & & $21.4 \%$ & & & $64.3 \%$ \\
\hline$p$-value & & & & 0.0248 & & \\
\hline
\end{tabular}

Note: NR, no response.

*The detected response was not statistically significant under EC correction.

In Table 4, we present the main findings regarding the peak $t$-statistical scores and the spatial profiles of the detected hemodynamic changes associated with innocuous stimuli. Using a similar detection process, we also observed an increase in the detection sensitivity of innocuous stimuli from $21.4 \%$ to $64.3 \%$ when the detection model was applied. However, the improvement in peak $t$-scores of the detected hemodynamic changes associated with innocuous stimuli $(p=0.0248)$ was less significant than that with noxious stimuli.

\section{Discussion}

\subsection{Summary of Findings and Potential Clinical Applications}

In the first part of this paper, we provided a preliminary assessment of the test-retest reliability of the fNIRS-measured aPFC response to noxious and innocuous stimuli. Based on the results from 14 healthy, male subjects, the hemodynamic changes associated with noxious stimuli seemed to have a higher reproducibility (i.e., with higher ICC and Spearman's correlation coefficient values) than innocuous stimuli in the medial portion of the aPFC. Moreover, we observed that responses recorded from the medial channels ( $\mathrm{C} 3$ and $\mathrm{C} 4)$ were also generally more reliable than those from the lateral channels ( $\mathrm{C} 2$ and $\mathrm{C} 5)$.
These findings are concordant with the findings reported in our previous papers ${ }^{6,7}$ that the hemodynamic response to painful stimuli was stronger than that to nonpainful stimuli and that the response was most strongly present in the medial portion of the aPFC. The overall good test-retest reliability of aPFC response to pain on a single subject provides the basis of employing a prerecorded pain response function to detect subsequent perception of painful events.

Based on these results, in the second part, we proposed a GLM-based detection model that detects the aPFC hemodynamic response to noxious and innocuous stimuli in individual subjects using prerecorded individualized HRFs. The detection sensitivity of the proposed model was estimated based on the 14 subjects and was compared with a standard GLM approach using a canonical HRF. We reported that using the prerecorded SHRFs significantly improved the detection sensitivity of pain response in 10 subjects with an increase in sensitivity from $14.3 \%$ to $64.3 \%$. In addition, the detection model also improved the sensitivity of detecting innocuous stimuli. However, the statistical significance of the improvement was lower.

Taken together, this study showed that fNIRS is a reliable tool for detecting the prefrontal response to pain and described a simple but practical method in applying fNIRS to perform 
rapid, robust pain detection, and evaluation. We believe that this approach can be easily implemented (as it only requires the addition of a brief prescan) and can potentially be used in various pain-related clinical applications, such as to monitor pain in patients unable to speak and to test the efficacy of pain treatment (e.g., opioid effect). An example of using this approach would be to test the level of analgesia on patients undergoing surgical treatment. While a prescan can be easily conducted on a patient preoperatively, the recorded pain-related hemodynamic response may then be used to detect and evaluate his/her perception of pain in the perioperative period.

\subsection{Comparison of Our Results with Those from Previous Studies}

FNIRS and BOLD-fMRI share a similar physiological basis in that they both measure hemoglobin concentration-related changes in the brain. In this study, we first evaluated the test-retest reliability of pain measures using fNIRS in a way similar to previous fMRI studies. ${ }^{31-33}$ With BOLD-fMRI, Quiton et al. ${ }^{31}$ observed deactivations in BOLD signals over the medial portion of the aPFC for heat pain and reported ICC values of 0.668 to 0.771 for the amplitude of the signal decrease. Upadhyay et al. ${ }^{33}$ focused primarily on the activation clusters over the prefrontal cortex following pain and obtained ICC values of 0.515 to 0.787 for the response magnitude. These results are generally comparable to the ICC values we estimated for fNIRS from the medial channels (ICCs for nadir/peak magnitude: 0.660 to 0.785 ).

Regarding pain detection, several studies (mostly based on fMRI, but also a few on fNIRS) have explored the feasibility of developing practical models in using brain signals to recognize painful events or to predict subjective pain intensities. ${ }^{34-37}$ In particular, models employing machine-learning techniques have been shown to yield promising results. For example, using fNIRS signals from the anterolateral prefrontal cortex (which mostly delineated an $\mathrm{HbO}$ increase following pain) and a support vector machine (SVM)-based technique, Pourshoghi et al. ${ }^{38}$ obtained an accuracy of up to $94 \%$ in classifying highpain and low-pain events. Despite the focus being on post-hoc painful event classification and clustering (rather than pain detection as in our study), their work is still remarkable in demonstrating the feasibility of using fNIRS as a useful tool in objective pain assessment. Karamzadeh et al. ${ }^{39}$ applied fNIRS in the classification of patients with traumatic brain injury and showed its potential in identifying brain markers of injury or disease. Using fMRI, Brown et al. ${ }^{34}$ reported $81 \%$ accuracy in distinguishing painful from nonpainful stimuli with an SVM approach. Another study by Wager et al. ${ }^{37}$ reported a pattern of fMRI activity associated with heat pain and showed high sensitivity and specificity (both above 90\%) in using the specific pattern to distinguish painful heat from nonpainful warmth, pain anticipation, and pain recall. These estimates of pain detection sensitivity with fMRI are much higher than the numbers reported in this study with fNIRS (64.3\% sensitivity). First, fMRI has better spatial resolution and is able to sample from deep structures of the brain, which allows for the delineation of a much more general pattern of pain signals involving multiple cortical and subcortical regions. For example, the neurologic signature used in Wager et al. ${ }^{37}$ included activities in the secondary somatosensory area, the cingulate cortex, the thalamus, the insula cortex, and the periaqueductal gray matter, which are the main regions reported to be reliably activated during experimental acute pain. ${ }^{32,33}$ On the other hand, fNIRS is able to reliably collect hemodynamic signals from the cortical surfaces, therefore limiting its ability to detect deep layer pain response. However, fNIRS has excellent robustness to accommodate clinical settings (e.g., in the operating room, bedside). In this study, we focused on the aPFC response. Advantages of putting NIRS optodes on the forehead include the ease of access and the ability to eliminate hair contaminations to obtain a high signal-to-noise ratio. ${ }^{7}$ Finally, it should be noted that machinelearning approaches usually require a large sample set to train classifiers to achieve a reasonable sensitivity and specificity. ${ }^{40}$ In this fNIRS study, however, we showed that the detection sensitivity of pain response could be significantly improved just by adding a short prerecorded session (a 3-min acquisition containing three noxious stimuli) prior to the actual noxious scan.

\subsection{Activation and Deactivation in the aPFC Associated with Nociception}

In this study, we focused on the hemodynamic response in the aPFC, also known as the frontopolar (FP) cortex, which corresponds with the Brodmann area 10. The role of the aPFC in pain perception and modulation has not been fully elucidated. Besides deactivations, some studies have also reported aPFC activations during or following painful/noxious stimulus (e.g., see Apkarian et al. ${ }^{41}$ for an early review), especially in the lateral portion of the aPFC. In this study, while a deactivation process was mostly observed in the medial aPFC, $\mathrm{HbO}$ increases (i.e., cortical activation) after electrical pain were also noticed, primarily from the lateral channels (e.g., on S2, S4, S5, S6, S7, S8, S10, and S11, see Fig. 3). Connectivity-based studies have proposed either two subregions (medial and FPm and lateral FPl ${ }^{42}$ or three subregions (FPm, FPl and orbital, and FPo) parcellation ${ }^{43}$ for the aPFC, with each subregion associated with different connections and functions: $\mathrm{FPl}$ - cognitive processing network; FPo-social emotional network, and FPm-the default mode network (DMN). Therefore, the activations that we observed in the lateral aPFC might reflect high-level processing and integration of the sensory information of pain, as well as the associated emotional processing. Moreover, previous work reported that the lateral aPFC was activated during opioid, ${ }^{44,45}$ stimulation, ${ }^{46,47}$ and placebo-induced ${ }^{48,49}$ analgesia, suggesting its role in the modulation of pain. On the other hand, deactivation of the medial aPFC following pain was often seen to be associated with other regions in the DMN..$^{15,50}$ The DMN is involved in interoception, self-referential evaluation and is generally deactivated when a task or salient event is presented. ${ }^{51,52}$ Indeed, Kucyi et al. ${ }^{53}$ reported that the paininduced DMN deactivation was attenuated when the subject's attention was distracted from pain. In support of complex interactions whereby pain may inhibit activities in frontal areas, research in a nonhuman animal model has revealed "glutamatergic afferents from the amygdala monosynaptically innervate GABAergic interneurons in the medial prefrontal cortex, which synapse on layer $\mathrm{V}$ pyramidal cells and control pyramidal cell output." 54

Conceptually, the above-mentioned studies provide a model for the aPFC response profile we observed on our subjects, i.e., activations in the lateral portion (related to sensory-discriminative, affective-motivational aspects of pain, and endogenous pain modulation) and deactivations in the medial portion (related to attentional aspect and other inhibitory processes). 
By simultaneously looking at the contrast maps generated with sHRFs from both medial and lateral channels, the proposed detection model is likely to combine these distinct cortical representations associated with pain in the detection, which might also potentially explain the significant sensitivity improvement over the standard GLM assuming only aPFC deactivations with a fixed shape.

\subsection{Limitations}

There are a few limitations related to this study including: (1) Sample size: as an assessment of test-retest reliability and pain detection sensitivity, this study was conducted on a relatively small subject sample size $(n=14)$. In particular, the detection set for each subject contained only three noxious stimuli and three innocuous stimuli. This might lower the statistical significance of the detected responses with the GLM. (2) Sampling brain region: this study focused exclusively on the aPFC response to pain. The detection sensitivity may be further increased when fNIRS signals can be reliably collected from both prefrontal and somatosensory regions. (3) Types of pain and sex differences: this study was restricted to the detection of electrical pain in male subjects. However, individuals have different pain thresholds or susceptibility to pain and analgesics, presumably based at least in part on the baseline "resting state" condition that may change with behaviors (e.g., sleep deprivation), genetic contributions, ${ }^{55,56}$ or sex. ${ }^{57}$ Further work incorporating various types of pain stimuli on both genders would be necessary to determine the usefulness of the proposed detection model. (4) Pain habituation: we did not observe significant habituation of pain signals in this study possibly due to the small number of stimuli, the short acquisition time, the small number of subjects involved, and a relatively long resting period between the two scans $(30 \mathrm{~min}$ ). However, we anticipate that severe habituation of pain response in other cases may have a negative impact on the test-retest reliability and the detection sensitivity.

\section{Conclusions}

In this work, we report that fNIRS has a reasonable reliability in detecting the hemodynamic response to noxious stimuli from the medial portion of the aPFC. Based on these results, we proposed a GLM-based model that employs prerecorded, personalized HRFs to detect pain and showed a significantly higher sensitivity in detecting a subject's pain perception using the proposed model than the standard GLM approach. This study supports that fNIRS may have the potential to become a clinically useful tool for accessing and evaluating pain and highlights the importance of performing careful modeling and individualized analysis in data processing. Further work on the improvements in data acquisition and the analytical methods (especially, to evaluate the detection specificity), as well as on validations with different types of pain stimuli on a larger number of both male and female subjects, might be beneficial.

\section{Appendix: Supplementary Data}

\section{A.1 Comparison of the Estimated sHRFs to Innocuous Stimuli with the cHRF}

We depict the sHRFs to innocuous stimuli from the medial prefrontal channels (Fig. 5) and the lateral channels (Fig. 6), respectively.
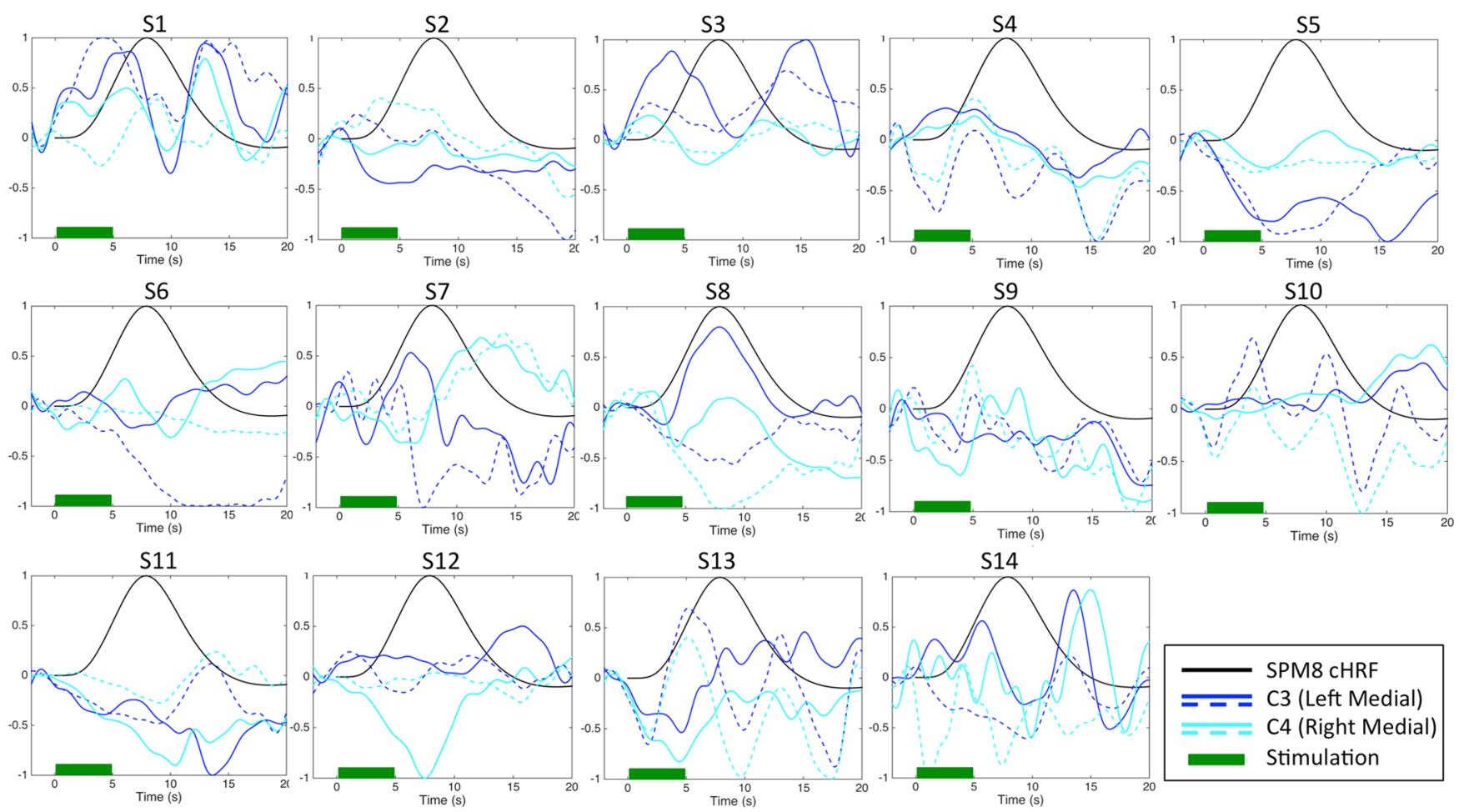

Fig. 5 Medial channels (C3 and C4): comparison of the SPM8 cHRF and the channel-wise sHRFs to innocuous stimuli estimated from the prescan (solid curves) and the detection scan (dashed curves). 

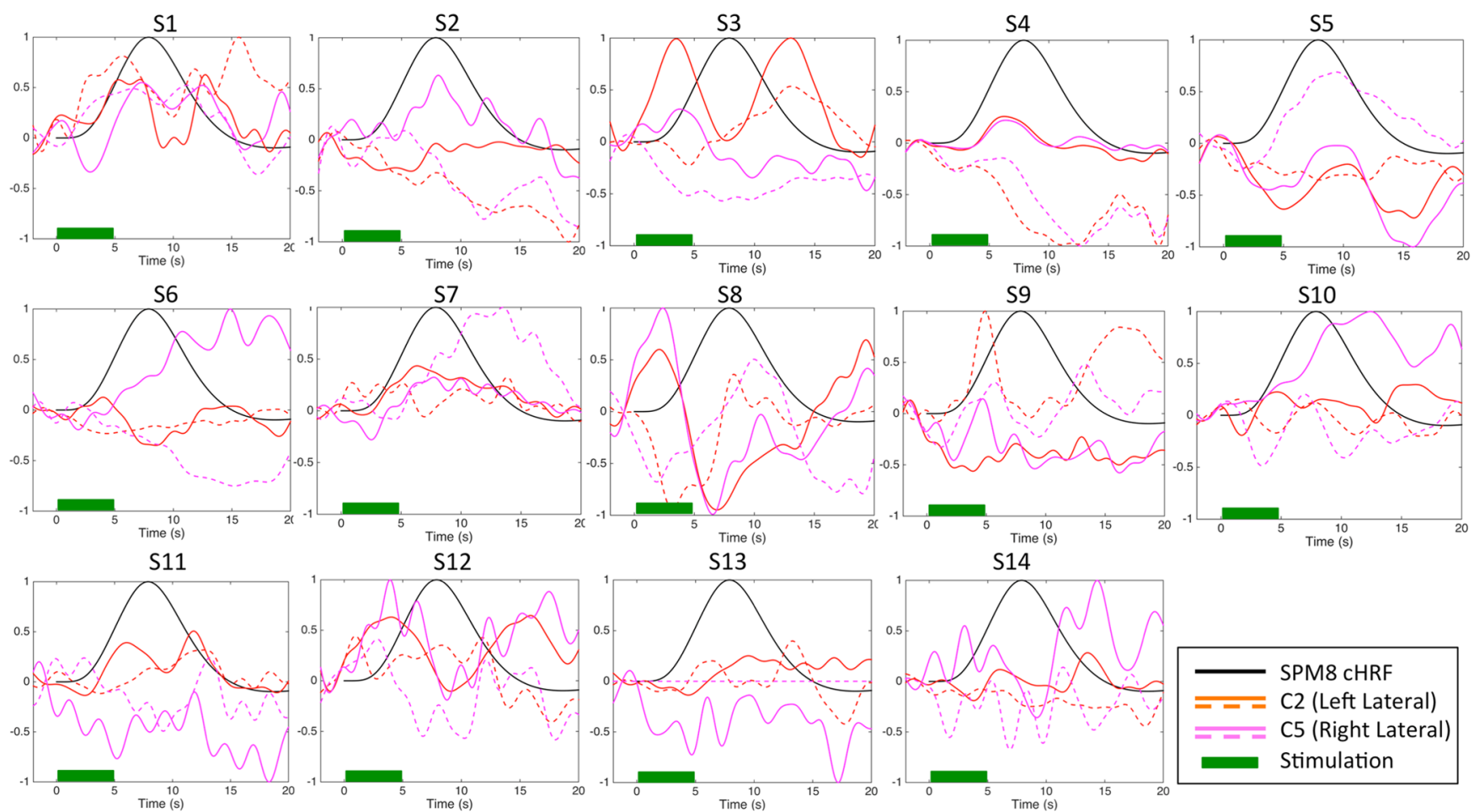

Fig. 6 Lateral channels (C2 and C5): comparison of the SPM8 cHRF and the channel-wise sHRFs to innocuous stimuli estimated from the prescan (solid curves) and the detection scan (dashed curves).

\section{A.2 Peak/Nadir Magnitudes and Latency of the Identified Main Response of the sHRFs}

Tables 5 and 6 present the peak/nadir magnitudes and latency of the identified main response of the estimated sHRFs of each subject from the prescan and the detection scan.

Table 5 Peak/nadir magnitudes and latency of the identified main response of the estimated sHRFs from the prescan (scan 1).

Scan 1

\begin{tabular}{|c|c|c|c|c|c|c|c|c|}
\hline \multirow{2}{*}{$\frac{\text { Peak amplitude }(\mu \mathrm{M})}{\text { Subject }}$} & \multicolumn{4}{|c|}{ Innocuous stimuli } & \multicolumn{4}{|c|}{ Noxious stimuli } \\
\hline & $\mathrm{C} 2$ & C3 & $\mathrm{C} 4$ & $\mathrm{C} 5$ & $\mathrm{C} 2$ & C3 & $\mathrm{C} 4$ & $\mathrm{C} 5$ \\
\hline 1 & 0.1515 & 0.2647 & 0.1510 & 0.1663 & -0.3255 & -0.5178 & -0.5804 & -0.3276 \\
\hline 2 & -0.1987 & -0.2887 & -0.0274 & 0.3723 & -0.3869 & -0.4486 & -0.2453 & 0.8949 \\
\hline 3 & 0.5696 & 0.7614 & -0.1939 & -0.1742 & -0.1758 & -0.8760 & -0.4152 & -0.4332 \\
\hline 4 & -0.0200 & 0.1602 & 0.0916 & 0.1304 & 0.2432 & -0.1021 & 0.0974 & 1.0168 \\
\hline 5 & -0.2526 & -0.3056 & -0.0917 & -0.1871 & 0.2146 & -0.0960 & 0.1570 & 0.3949 \\
\hline 6 & -0.1553 & -0.0888 & -0.1061 & 0.4075 & 0.0474 & -0.1468 & -0.1673 & -0.0663 \\
\hline 7 & 0.1725 & -0.0150 & -0.0483 & 0.1234 & 0.0975 & -0.3911 & -0.1847 & 0.1474 \\
\hline 8 & -0.3315 & 0.3190 & -0.0789 & -0.2935 & 0.2474 & -0.2287 & -0.4880 & 0.3409 \\
\hline 9 & -0.1744 & -0.0626 & -0.1140 & -0.1453 & -0.4472 & -0.0890 & -0.2806 & -0.2290 \\
\hline 10 & 0.1218 & 0.0489 & 0.0647 & 0.7397 & 0.5553 & -0.1007 & -0.0887 & -0.5632 \\
\hline 11 & 0.2765 & -0.4430 & -0.4062 & -0.4556 & 0.7744 & 1.2092 & 0.3838 & 0.7547 \\
\hline 12 & 0.3385 & 0.1685 & -0.6963 & 0.3930 & -0.5713 & -0.2220 & -0.6419 & -0.3199 \\
\hline 13 & 0.1608 & -0.2657 & -0.5263 & NR & -0.3889 & -0.6440 & -0.8834 & NR \\
\hline 14 & -0.0612 & -0.0788 & -0.1194 & -0.0601 & -0.0024 & -0.0067 & -0.3847 & -0.1420 \\
\hline
\end{tabular}


Table 5 (Continued).

Scan 1

\begin{tabular}{|c|c|c|c|c|c|c|c|c|}
\hline \multirow{2}{*}{$\begin{array}{l}\text { Peak latency (s) } \\
\text { Subject }\end{array}$} & \multicolumn{4}{|c|}{ Innocuous stimuli } & \multicolumn{4}{|c|}{ Noxious stimuli } \\
\hline & $\mathrm{C} 2$ & C3 & $\mathrm{C} 4$ & C5 & $\mathrm{C} 2$ & $\mathrm{C} 3$ & $\mathrm{C} 4$ & C5 \\
\hline 1 & 7.2 & 6.4 & 6.16 & 7.32 & 10.28 & 10.76 & 10.64 & 12.68 \\
\hline 2 & 5.84 & 4.16 & 7.8 & 8.12 & 10.08 & 10.04 & 9.96 & 6.04 \\
\hline 3 & 3.56 & 3.92 & 6.72 & 9.84 & 9.08 & 10.92 & 6.96 & 8.96 \\
\hline 4 & 11.28 & 2.8 & 5.2 & 6.44 & 5.72 & 12.76 & 12.56 & 7.28 \\
\hline 5 & 5.08 & 5.88 & 5.24 & 3.84 & 8.4 & 5.52 & 9.2 & 7.92 \\
\hline 6 & 8.56 & 6.72 & 10.04 & 14.92 & 7.76 & 6.32 & 10.2 & 12.04 \\
\hline 7 & 6.34 & 9.18 & 6.44 & 7.74 & 5.38 & 10.22 & 6.02 & 9.16 \\
\hline 8 & 6.92 & 7.9 & 4.26 & 6.56 & 4.32 & 2.96 & 7.94 & 10.18 \\
\hline 9 & 3.9 & 10.24 & 12.56 & 9.1 & 8.14 & 10.82 & 12.62 & 10.24 \\
\hline 10 & 4.68 & 10.8 & 8.38 & 12.44 & 5.74 & 13.16 & 11.1 & 6 \\
\hline 11 & 11.84 & 13.62 & 10.42 & 5.22 & 11.48 & 10.36 & 10.66 & 10.92 \\
\hline 12 & 4.04 & 4 & 7.52 & 3.94 & 6.9 & 8.06 & 8.68 & 9.72 \\
\hline 13 & 9.52 & 4.6 & 4.42 & NR & 10.72 & 6.88 & 7.34 & NR \\
\hline 14 & 3.82 & 9.3 & 9.92 & 9.22 & 4.82 & 4.56 & 4 & 6.1 \\
\hline
\end{tabular}

Note: NR, no response.

Table 6 Peak/nadir magnitudes and latency of the identified main response of the estimated sHRFs from the detection scan (scan 2).

Scan 2

\begin{tabular}{|c|c|c|c|c|c|c|c|c|}
\hline \multirow{2}{*}{$\frac{\text { Peak amplitude }(\mu \mathrm{M})}{\text { Subject }}$} & \multicolumn{4}{|c|}{ Innocuous stimuli } & \multicolumn{4}{|c|}{ Noxious stimuli } \\
\hline & $\mathrm{C} 2$ & C3 & $\mathrm{C} 4$ & C5 & $\mathrm{C} 2$ & C3 & $\mathrm{C} 4$ & C5 \\
\hline 1 & 0.2673 & 0.3415 & 0.0906 & 0.1545 & -0.3787 & -0.4956 & -0.3110 & -0.2907 \\
\hline 2 & -0.3121 & 0.0066 & 0.2196 & -0.5454 & 0.7960 & 0.4599 & 0.3591 & 0.2160 \\
\hline 3 & -0.0806 & 0.1474 & -0.1131 & -0.3800 & -0.3751 & -1.0388 & -0.5632 & -0.3292 \\
\hline 4 & -0.7366 & -0.0253 & 0.1493 & -0.1480 & 0.1727 & -0.4147 & -0.3159 & 0.1771 \\
\hline 5 & -0.1293 & -0.3575 & -0.1112 & 0.2846 & -0.1416 & -0.2044 & -0.1491 & 0.1739 \\
\hline 6 & -0.1138 & -0.4901 & -0.1330 & -0.3821 & -0.5321 & -0.2217 & -0.0129 & -0.2596 \\
\hline 7 & 0.0701 & -0.1288 & -0.0272 & 0.4082 & -0.2904 & -0.5818 & -0.2200 & 0.3911 \\
\hline 8 & -0.2933 & -0.2287 & -0.4337 & -0.2090 & -0.3828 & -0.0112 & -0.0687 & -0.1248 \\
\hline 9 & 0.1811 & -0.1088 & -0.0842 & -0.0417 & 0.2401 & -0.0279 & -0.1766 & 0.0819 \\
\hline 10 & -0.0871 & 0.1778 & -0.3721 & -0.2298 & -0.2127 & 0.0515 & -0.0768 & -0.2211 \\
\hline 11 & 0.2160 & -0.2270 & -0.1140 & -0.2903 & -0.6744 & 0.8737 & 0.3282 & -0.5784 \\
\hline 12 & 0.1183 & 0.1388 & -0.0608 & 0.1317 & -0.4533 & -0.5097 & -0.3494 & 0.2950 \\
\hline 13 & 0.0917 & 0.3783 & -0.5664 & NR & 0.1748 & -0.4844 & -1.0006 & NR \\
\hline 14 & -0.1660 & -0.2349 & -0.2662 & -0.2276 & -0.0822 & -0.1515 & -0.1681 & -0.0494 \\
\hline
\end{tabular}


Table 6 (Continued).

Scan 2

\begin{tabular}{|c|c|c|c|c|c|c|c|c|}
\hline \multirow{2}{*}{$\begin{array}{l}\text { Peak latency (s) } \\
\text { Subject }\end{array}$} & \multicolumn{4}{|c|}{ Innocuous stimuli } & \multicolumn{4}{|c|}{ Noxious stimuli } \\
\hline & $\mathrm{C} 2$ & C3 & $\mathrm{C} 4$ & C5 & $\mathrm{C} 2$ & $\mathrm{C} 3$ & $\mathrm{C} 4$ & C5 \\
\hline 1 & 5.68 & 4.2 & 8.84 & 11.2 & 8.4 & 8.44 & 8.08 & 14 \\
\hline 2 & 6.36 & 5.92 & 3.32 & 12 & 10.48 & 10.72 & 10.24 & 9.64 \\
\hline 3 & 4.6 & 7.84 & 7.88 & 7.36 & 9.24 & 9.32 & 7.72 & 9.88 \\
\hline 4 & 11.08 & 5.2 & 5.16 & 6.08 & 4.8 & 13.2 & 13.24 & 5.48 \\
\hline 5 & 12 & 6.64 & 4.36 & 9.48 & 8.68 & 8.2 & 9.64 & 4.4 \\
\hline 6 & 4.4 & 10 & 14.24 & 15.24 & 7.04 & 10 & 7.36 & 10.2 \\
\hline 7 & 5.18 & 7.18 & 5.06 & 13.52 & 9.04 & 11.96 & 10.96 & 12.86 \\
\hline 8 & 3.18 & 9.06 & 8.1 & 1.88 & 12.34 & 3.72 & 9.34 & 2.26 \\
\hline 9 & 4.92 & 11.5 & 11.06 & 9.42 & 3.08 & 8.3 & 10.1 & 9.26 \\
\hline 10 & 11.2 & 3.92 & 13.02 & 3.42 & 5.64 & 9.04 & 12 & 5.82 \\
\hline 11 & 12.78 & 8.9 & 9.1 & 9.22 & 8.74 & 12.36 & 12.28 & 5.42 \\
\hline 12 & 11.82 & 11.28 & 2.46 & 2.84 & 6.96 & 7.3 & 6.88 & 4.06 \\
\hline 13 & 6.42 & 5.18 & 9.68 & NR & 9.92 & 9.4 & 9.9 & NR \\
\hline 14 & 8.58 & 9.56 & 7.4 & 6.92 & 11.1 & 7.9 & 11.18 & 4.86 \\
\hline
\end{tabular}

Note: NR, no response.

\section{Disclosures}

David A. Boas is an inventor of the technology licensed to TechEn, Inc., which is a company that focuses on providing solutions to noninvasive optical brain imaging. David A. Boas's interests were reviewed and are managed by Massachusetts General Hospital and Partners HealthCare in accordance with their conflict of interest policies. All other authors have no conflicts of interest to declare.

\section{Acknowledgments}

This study was supported by the National Institutes of Health (NIH) and the National Institute of General Medical Sciences (NIGMS) Grant No. NIH-NIGMS 1-R01-GM104986.

\section{References}

1. H. Obrig, "NIRS in clinical neurology: a 'promising' tool?" NeuroImage 85(Part 1), 535-546 (2014).

2. M. Ferrari and V. Quaresima, "A brief review on the history of human functional near-infrared spectroscopy (fNIRS) development and fields of application," NeuroImage 63, 921-935 (2012).

3. D. T. Delpy and M. Cope, "Quantification in tissue near-infrared spectroscopy," Philos. Trans. R. Soc. B Biol. Sci. 352, 649-659 (1997).

4. F. F. Jöbsis, "Noninvasive, infrared monitoring of cerebral and myocardial oxygen sufficiency and circulatory parameters," Science 198, 1264 1267 (1977).

5. S. Lloyd-Fox, A. Blasi, and C. E. Elwell, "Illuminating the developing brain: the past, present and future of functional near infrared spectroscopy," Neurosci. Biobehav. Rev. 34, 269-284 (2010).

6. M. A. Yücel et al., "Specificity of hemodynamic brain responses to painful stimuli: a functional near-infrared spectroscopy study," Sci. Rep. 5, 9469 (2015).
7. C. M. Aasted et al., "Frontal lobe hemodynamic responses to painful stimulation: a potential brain marker of nociception," PLoS One $\mathbf{1 1}$ e0165226 (2016)

8. L. Holper et al., "Physiological effects of mechanical pain stimulation at the lower back measured by functional near-infrared spectroscopy and capnography," J. Integr. Neurosci. 13, 121-142 (2014).

9. S. Sakuma et al., "Experimental pain in the gingiva and its impact on prefrontal cortical hemodynamics: a functional near-infrared spectroscopy study," Neurosci. Lett. 575, 74-79 (2014).

10. L. Becerra et al., "Brain measures of nociception using near-infrared spectroscopy in patients undergoing routine screening colonoscopy," Pain 157, 840-848 (2016).

11. B. D. Kussman et al., "Capturing pain in the cortex during general anesthesia: near infrared spectroscopy measures in patients undergoing catheter ablation of arrhythmias," PLoS One 11, e0158975 (2016).

12. J. Kong et al., "Exploring the brain in pain: activations, deactivations and their relation," Pain 148, 257-267 (2010).

13. F. Lui et al., "Touch or pain? Spatio-temporal patterns of cortical fMRI activity following brief mechanical stimuli," Pain 138, 362-374 (2008).

14. H. Gündel et al., "Altered cerebral response to noxious heat stimulation in patients with somatoform pain disorder," Pain 137, 413-421 (2008).

15. M. L. Loggia et al., "Disentangling linear and nonlinear brain responses to evoked deep tissue pain," Pain 153, 2140-2151 (2012).

16. C. Gélinas et al., "Toward a new approach for the detection of pain in adult patients undergoing cardiac surgery: near-infrared spectroscopy: a pilot study," Heart Lung J. Acute Crit. Care 39, 485-493 (2010).

17. Z. Barati et al., "Hemodynamic response to repeated noxious cold pressor tests measured by functional near infrared spectroscopy on forehead," Ann. Biomed. Eng. 41, 223-237 (2013).

18. C.-H. Lee et al., "Analysis for distinctive activation patterns of pain and itchy in the human brain cortex measured using near infrared spectroscopy (NIRS)," PLoS One 8, e75360 (2013).

19. A. Yennu et al., "Prefrontal hemodynamic mapping by functional nearinfrared spectroscopy in response to thermal stimulations over three body sites," Neurophotonics 3, 045008 (2016). 
20. M. M. Monti, "Statistical analysis of fMRI time-series: a critical review of the GLM approach," Front. Hum. Neurosci. 5, 28 (2011).

21. M. A. Yücel et al., "Mayer waves reduce the accuracy of estimated hemodynamic response functions in functional near-infrared spectroscopy," Biomed. Opt. Express 7, 3078-3088 (2016).

22. T. J. Huppert et al., "HomER: a review of time-series analysis methods for near-infrared spectroscopy of the brain," Appl. Opt. 48, D280-D298 (2009).

23. L. Gagnon et al., "Improved recovery of the hemodynamic response in diffuse optical imaging using short optode separations and state-space modeling," NeuroImage 56, 1362-1371 (2011).

24. P. E. Shrout and J. L. Fleiss, "Intraclass correlations: uses in assessing rater reliability," Psychol. Bull. 86, 420-428 (1979).

25. K. Peng et al., "fNIRS-EEG study of focal interictal epileptiform discharges," Epilepsy Res. 108, 491-505 (2014).

26. K. J. Friston et al., Statistical Parametric Mapping: The Analysis of Functional Brain Images, Academic Press, London (2006).

27. J. C. Ye et al., "NIRS-SPM: statistical parametric mapping for nearinfrared spectroscopy," Neurolmage 44, 428-447 (2009).

28. S. Tak and J. C. Ye, "Statistical analysis of fNIRS data: a comprehensive review," Neurolmage 85(Pt 1), 72-91 (2014).

29. H. Li, S. Tak, and J. C. Ye, "Lipschitz-Killing curvature based expected Euler characteristics for p-value correction in fNIRS," J. Neurosci. Methods 204, 61-67 (2012).

30. R. Rathakrishnan et al., "BOLD signal changes preceding negative responses in EEG-fMRI in patients with focal epilepsy," Epilepsia 51, 1837-1845 (2010).

31. R. L. Quiton et al., "Intersession reliability of fMRI activation for heat pain and motor tasks," NeuroImage Clin. 5, 309-321 (2014).

32. J. E. Letzen et al., "Test-retest reliability of pain-related brain activity in healthy controls undergoing experimental thermal pain," J. Pain 15, 1008-1014 (2014).

33. J. Upadhyay et al., "Test-retest reliability of evoked heat stimulation BOLD fMRI," J. Neurosci. Methods 253, 38-46 (2015).

34. J. E. Brown et al., "Towards a physiology-based measure of pain: patterns of human brain activity distinguish painful from non-painful thermal stimulation," PLoS One 6 (2011).

35. J. K. Brodersen et al., "Decoding the perception of pain from fMRI using multivariate pattern analysis," NeuroImage 63, 1162-1170 (2012).

36. A. Marquand et al., "Quantitative prediction of subjective pain intensity from whole-brain fMRI data using Gaussian processes," NeuroImage 49, 2178-2189 (2010).

37. T. D. Wager et al., "An fMRI-based neurologic signature of physical pain," N. Engl. J. Med. 368, 1388-1397 (2013).

38. A. Pourshoghi, I. Zakeri, and K. Pourrezaei, "Application of functional data analysis in classification and clustering of functional near-infrared spectroscopy signal in response to noxious stimuli," J. Biomed. Opt. 21, 101411 (2016).

39. N. Karamzadeh et al., "A machine learning approach to identify functional biomarkers in human prefrontal cortex for individuals with traumatic brain injury using functional near-infrared spectroscopy," Brain Behav. 6, e00541 (2016).

40. C. Beleites et al., "Sample size planning for classification models," Anal. Chim. Acta 760, 25-33 (2013).

41. A. V. Apkarian et al., "Human brain mechanisms of pain perception and regulation in health and disease," Eur. J. Pain Lond. Engl. 9, 463-463 (2005).

42. M. Moayedi et al., "Connectivity-based parcellation of the human frontal polar cortex," Brain Struct. Funct. 220, 2603-2616 (2015).

43. H. Liu et al., "Connectivity-based parcellation of the human frontal pole with diffusion tensor imaging," J. Neurosci. Off. J. Soc. Neurosci. 33, 6782-6790 (2013).

44. K. J. Wagner et al., "Dose-dependent regional cerebral blood flow changes during remifentanil infusion in humans: a positron emission tomography study," Anesthesiology 94, 732-739 (2001).

45. T. Sprenger et al., "Opioidergic activation in the medial pain system after heat pain," Pain 122, 63-67 (2006).

46. H. Kishima et al., "Modulation of neuronal activity after spinal cord stimulation for neuropathic pain; H215O PET study," Neurolmage 49, 2564-2569 (2010).
47. S. H. Ohn et al., "Neural correlates of the antinociceptive effects of repetitive transcranial magnetic stimulation on central pain after stroke," Neurorehabil. Neural Repair 26, 344-352 (2012).

48. P. Petrovic et al., "A prefrontal non-opioid mechanism in placebo analgesia," Pain 150, 59-65 (2010).

49. M. Amanzio et al., "Activation likelihood estimation meta-analysis of brain correlates of placebo analgesia in human experimental pain," Hum. Brain Mapp. 34, 738-752 (2013).

50. L. van Oudenhove et al., "Cortical deactivations during gastric fundus distension in health: visceral pain-specific response or attenuation of 'default mode' brain function? A H2 15O-PET study," Neurogastroenterol. Motil. Off. J. Eur. Gastrointest. Motil. Soc. 21, 259-271 (2009).

51. L. Q. Uddin et al., "Functional connectivity of default mode network components: correlation, anticorrelation, and causality," Hum. Brain Mapp. 30, 625-637 (2009).

52. D. Sridharan, D. J. Levitin, and V. Menon, "A critical role for the right fronto-insular cortex in switching between central-executive and default-mode networks," Proc. Natl. Acad. Sci. U. S. A. 105, 12569 12574 (2008).

53. A. Kucyi, T. V. Salomons, and K. D. Davis, "Mind wandering away from pain dynamically engages antinociceptive and default mode brain networks," Proc. Natl. Acad. Sci. U. S. A. 110, 18692-18697 (2013).

54. G. Ji et al., "Cognitive impairment in pain through amygdala-driven prefrontal cortical deactivation," J. Neurosci. Off. J. Soc. Neurosci. 30, 5451-5464 (2010).

55. R. Fillingim et al., "Genetic contributions to pain: a review of findings in humans," Oral Dis. 14, 673-682 (2008).

56. Z.-Y. Ren et al., "The impact of genetic variation on sensitivity to opioid analgesics in patients with postoperative pain: a systematic review and meta-analysis," Pain Phys. 18, 131-152 (2015).

57. E. A. Moulton et al., "Sex differences in the cerebral BOLD signal response to painful heat stimuli," Am. J. Physiol. 291, R257-R267 (2006).

Ke Peng received his BSc degree in automation from Tsinghua University, China, his MASc degree in computer engineering from Ecole Polytechnique, University of Montreal, Canada, and his PhD in biomedical engineering from Ecole Polytechnique, University of Montreal, Canada. He is now a postdoc research fellow in the Pain and Analgesia Imaging Neuroscience (PAIN) Research Group at Boston Children's Hospital and Harvard Medical School. His current research interests pertain to the application of optical neuroimaging techniques in various clinical settings including perioperative pain assessment, epilepsy monitoring, and intensive care.

Meryem A. Yücel received her $\mathrm{PhD}$ in biomedical engineering from Biomedical Engineering Institute, Boğaziçi University, İstanbul, where she majored in chemical engineering at Boğaziçi University, İstanbul. Currently, she is an instructor at Harvard Medical School at the Athinoula A. Martinos Center for biomedical imaging and an assistant in biomedical engineering at Massachusetts General Hospital. Her research interests include neurovascular coupling and brain imaging using various modalities.

Christopher M. Aasted studied engineering at Colorado School of Mines before receiving his MS degree in mechatronic systems engineering and his PhD in engineering from the University of Denver. His research has focused on intelligent control systems and other machine-learning applications in both biomedical signal processing and unmanned aerial systems. He was an instructor at Harvard Medical School and a staff scientist at Boston Children's Hospital.

Sarah C. Steele graduated from the University of Mississippi in 2015 with a bachelor of science in biology. Currently, she is a research assistant in the PAIN Research Group at Boston Children's Hospital. She is involved in several projects using functional near-infrared spectroscopy (fNIRS) for the purpose of detecting brain activity associated with perioperative pain processing.

David A. Boas received his $\mathrm{PhD}$ in physics from the University of Pennsylvania. He is now a professor at Boston University, Radiology Department, Massachusetts General Hospital and Harvard Medical School. His research focuses on developing optical methods to 
study cerebral physiology and pathophysiology focusing on studying brain function, oxygen delivery, and consumption. He is the founding president of the society for fNIRS and the founding editor in chief of Neurophotonics.

David Borsook received his MD degree and PhD from the University of Witwatersrand, South Africa. He is a neurologist and neurobiologist by training. Currently, he directs the Center for Pain and the Brain and consults for a number of pharmaceutical and biotechnology companies in the fields of pain, analgesics, and imaging. $\mathrm{He}$ is a professor at Harvard Medical School and has appointments at McLean Hospital (psychiatry), Massachusetts General Hospital (radiology/psychiatry), and Boston Children's Hospital (anesthesiology/radiology).

Lino Becerra received his $\mathrm{PhD}$ in physics from the University of Illinois Urbana-Champaign. He studied physics at the Universidad Nacional de Ingenieria in Peru. His research has focused on the understanding of pain processing in the brain through neuroimaging techniques, including diffuse optical imaging and magnetic resonance. $\mathrm{He}$ is an associate professor at the Harvard Medical School and codirector of the Center for Pain and the Brain. 